\title{
Social Media-Addiction: Personality traits and human biology in the Facebook-age
}

Ulrich Kutschera*

Institute of Biology, University of Kassel, Germany \& The Systems Biology Group, Inc., Palo Alto, CA 94306, USA

On January 14, 2019, India Today reported that "Facebook addicts" are bad at making decisions because they think like those who are dependent on drugs, such as cocaine or heroin [1]. In this article, a study published in the Journal of Behavior Addiction is summarized, wherein Facebook usage was evaluated based on the "BFA-scale". This 6-score-measure ("Bergen Facebook Addiction Scale") can reveal the severity of Facebook addiction rather accurately. In addition, study subjects were also tested using the "Iowa Gambling Task" (IGT) to evaluate their real-life decision-making ability. As expected, those who perform better used social media less.

In this Comment, I review recent studies that revealed the hidden dangers that "digital footprints" may pose. First, the Five-Factor-Model of Personality is briefly discussed, and then facts about Facebook are summarized that are relevant to the disturbing report on the addictive potential of this social media outlet.

\section{The Five-Factor-Model of personality}

Dr. Michal Kosinski (Graduate School of Business, Stanford University, California) (Figure 1) has done research on human behavior and personality traits in Warsaw (Poland), Cambridge (United Kingdom) and California (USA). He has observed major differences in the expression of personality related to cultural dissimilarities between people in Europe and the USA. The same behavior can have different meanings for different people. In California, for instance, openmindedness, or a liberal attitude, is much more frequently expressed in the general population than, for instance, in the UK or Poland. Another

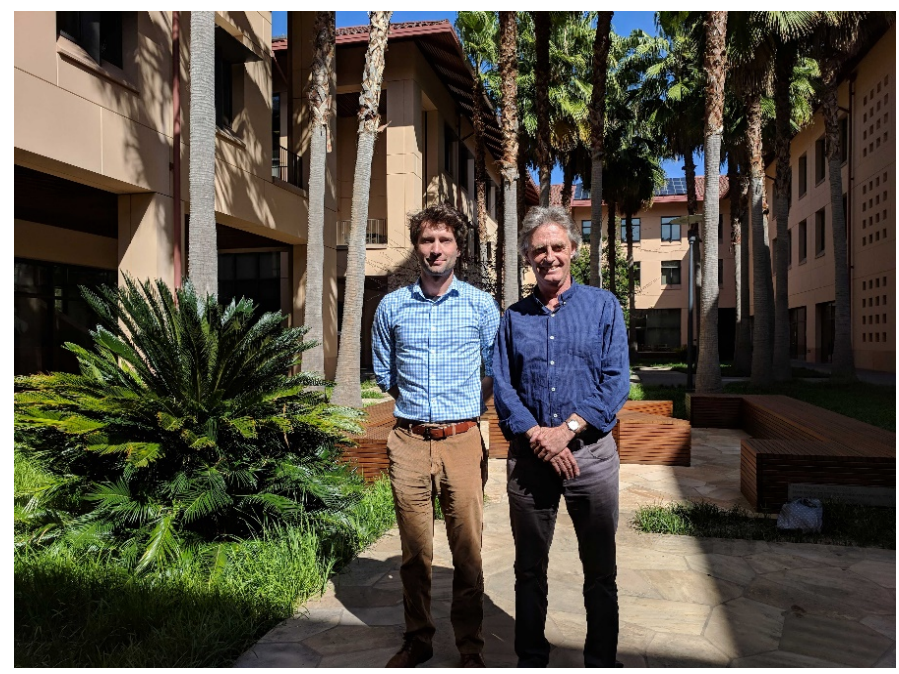

Figure 1. Professors Michal Kosinski and Ulrich Kutschera in the central yard of the Graduate School of Business, Stanford University example relates to religiosity; in San Francisco, Catholic churches celebrate mass different from their Polish counterparts. The following presentation of facts and data on social media addiction is based on a discussion between Dr. Kosinski and the author that took place on April 24, 2018 at Stanford University.

The "Five-Factor-Model of Personality", i.e. Openness, Conscientiousness, Extraversion, Agreeableness, and Neuroticism (OCEAN) is reminiscent to the "Big Five Mass extinction events" in evolutionary biology [2]. However, in evolutionary science, there are alternative interpretations of the fossil record. For instance, some ecologists argue that the "Sixth Mass extinction" is already in action. Based on this analogy, it is likely that other models exist that may challenge the Five-Factor-concept, notably with respect to "negative emotions (moodyness)". However, despite attempts to classify (or describe) personality differently, the 5-dimensional model seems to be the most widely accepted one. Before the "Big-Five", there were competing models describing many personality features. However, factor- and cluster-analyses of a multitude of personality models regularly boiled down to 5 dimensions accurately summarizing human behavior. Adopting this universally accepted scheme enabled personality researchers to communicate more efficiently. We do not think that another model will be established anytime soon. Concerning the tendency to experience "negative emotions", this is a part of the five-factor model, namely the traits of "Neuroticism" and "Openness to Experience" (Figure 2).

\section{Facebook-likes: race, gender and sexual orientation}

Six years ago (April 2013), Kosinski, et al. have published a remarkable PNAS-paper, entitled "Private traits and attributes are predictable from digital records of human behavior" [3]. This analysis was based on 58.000 Facebook-Likes; the average age of the participants was 25 years. The general response to this article was, to the surprise of the authors, overwhelming: according to Almetrics, it was the secondmost impactful paper published that year and has been cited nearly 1200 times in the last 5 years. In many ways, we think that Almetrics is superior to classical measures, such as Impact Factor and h-index. It can be described as a generalization of paper-level metrics and may be traced back to the \#almetrics hashtag.

We will first discuss the three personal attributes with the highest prediction accuracy, deduced from digital records: Ethnicity, also

${ }^{*}$ Correspondence to: $\mathrm{U}$ Kutschera, Professor of Biology, University of Kassel, Germany, E-Mail: kut@uni-kassel.de

Received: February 13, 2019; Accepted: March 06, 2019; Published: March 08 2019 


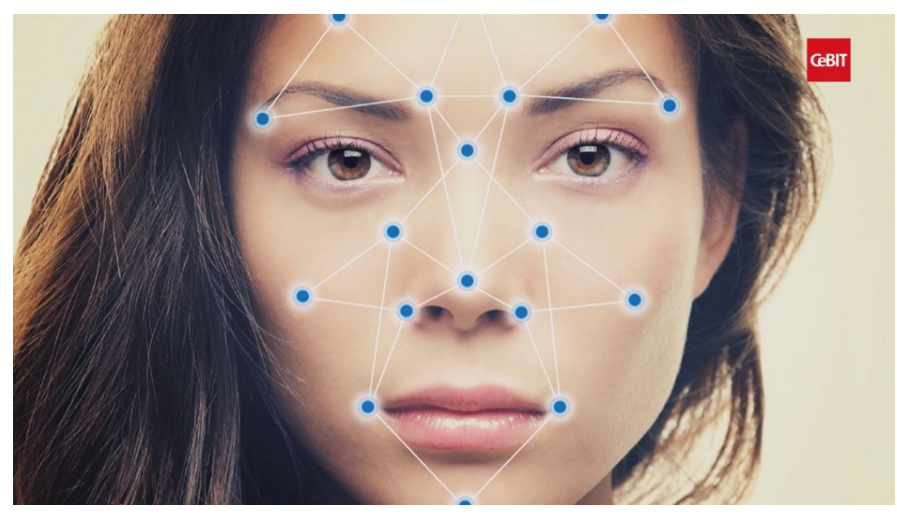

Figure 2. Face recognition using Artificial Intelligence. Image taken from Keynote: "The End of Privacy”, Dr. Michal Kosinski (24.03.2017, CeBIT Global Conference)

called Race (Caucasian vs. African American): $95 \%$; Gender (male vs. female): $93 \%$; and Sexual orientation (gay vs. straight in males): $88 \%$. It should be stressed that some social scientists argue ethnicity is only a social construct without biological basis. We think that there is nothing wrong with studying the social aspects of ethnicity. It is irrefutable, however, that it has a genetic and biological basis. Biological differences clearly exist, there are dark-pigmented and white Americans, and these differences are independent of the corresponding culture. In our sample of 7000 participants, about $73 \%$ were Caucasian, $14 \%$ African Americans, and $13 \%$ others. Hence, white Americans clearly outnumber other ethnicities with respect to Facebook-use [3].

Concerning gender-distribution, among over 57000 Facebookusers analyzed in this study, the majority were female (62\%), and only $38 \%$ male; the prediction accuracy was close to $93 \%$, almost as high as that concerning ethnicity. The explanation for this phenomenon is as follows. Research has shown that women use Facebook more frequently than men, i.e., the gender-ratio is not 50: 50, but about 60: 40. Moreover, data show that women spend more time on using Facebook than men, at least by $+20 \%$. The reason for this gender-gap is simple: women socialize more than men, and this has been confirmed by many independent studies in evolutionary psychology.

Next, sexual orientation in males, i.e., gay vs. straight, was analyzed, a personality trait detectable with an accuracy of $88 \%$ [3]. We interpret this finding as follows. Gay and straight individuals express sufficiently different behavior in the "online environment" to enable an accurate classification. Recently, more work was done on this subject - it seems that those differences expand also to the profile pictures that they post online, as well as to their facial features. This issue, nature vs. nurture with respect to sexual orientation, will be discussed below.

We will now take a look at the political views of Facebook-users. The prediction accuracy (Democrats vs. Republicans) was $85 \%$ a remarkable result [3]. An explanation may be given as follows. Behaviors and preferences of Democrats and Republicans, also those expressed in the online environment, are sufficiently different to allow algorithms to distinguish between them with high accuracy. In this study, the average age of participants was 25 years - about $65 \%$ were Liberals (Democrats), and only ca. $35 \%$ Conservatives (Republicans). The older people get, the more conservative political views emerge in their mind. Hence, an age-bias must be taken into account here.

Religion is another hot topic that Facebook-users provide to the general audience. Kosinski, et al. [3] yielded a prediction accuracy of
$82 \%$, based on your analysis of Facebook-Likes. An explanation may be as follows. Among the ca. 18.800 Facebook-users that participated in this study, about $90 \%$ were Christians and only $10 \%$ Muslims. This may reflect the fact that, in the US, Muslims represent a minority of the population (ca. $1 \%$ ). More research is necessary to find out why religious affiliation matters so much when it comes to Facebook use.

\section{Political propaganda and personalized communication}

Two years ago, another PNAS-article appeared in the section "Social Sciences", entitled "Psychological targeting as an effective approach to digital mass persuasion" [4]. This work provokes the following question discussed here. The German dictator and mass murder Adolf Hitler (1889-1945) used, during the 1930s, the Radio to reach millions of Germans for indoctrination with his horrendous Nazi-ideology. What technology would he use today, in the Facebookage? We suggest the following answer. Nazi-area propaganda was targeted on a city or country-level. Today, marketers can target their messages on a level of an individual. Current-day politicians, including our US-President, Donald J. Trump, but also major government officials of other parties and in other countries, employ this strategy. If Hitler were alive today, he would surely misuse Facebook and other social networks to distribute his despicable Nazi-propaganda. In their 2015 paper, the research team has outlined the danger of a potential misuse of digital mass persuasion [4].

\section{Gender-specific personality traits}

In a third PNAS-paper entitled "Computer-based personality judgements are more accurate than those made by humans", evidence was found for the objectivity and usefulness of the "Big-Five-Model of Personality" [5]. Notably, the researchers discovered that Computers are better than humans at predicting personality because of noise elimination. In addition, using these computer tools, one can predict the future behavior of humans. A key question is whether or not the five personality dimensions (OCEAN) are gender-specific.

Surprisingly, it was found that there are some differences between the sexes [5]. Women seem to be characterized by a higher level of conscientiousness, which may be driven both by nature and culture [5]. This has remarkable real-life consequences. In the American education system, girls increasingly outperform boys, who are less conscientious, on average. In the long term, this effect can lead to problems, such as a cohort of frustrated males, under-performing on the job market. Some commentators already talk about the "boy crisis" in the USA, another important question is whether or not these personality dimensions are genetically heritable and stable over developmental time of the individual. There is convincing evidence that personality traits are indeed heritable over subsequent generations and remain relatively constant during development of the corresponding person - from childhood to old age.

\section{Homosexuality and feminine vs. masculine faces}

Last year (2018), Wang and Kosinski published a controversial paper in the Journal of Personality and Social Psychology entitled "Deep Neural Networks are more accurate than humans at detecting sexual orientation from facial images" [6]. Their key finding was that they were able to document that gay men have, on average, more feminine faces than straight men. On the other hand, typical lesbians display more masculine features, compared to straight women [2]. They used Deep Neural Networks (DNN), i.e., computational models, or algorithms, inspired by the structure and function of animal brains. 
DNNs are composed of layers of artificial neurons (analogous to biological neurons) that process information, and establish connections (analogous to synapses).

This most recent study relates to the 2013 PNAS-paper referred to above, wherein a remarkable Facebook-Likes-prediction accuracy for gay vs. straight men of $88 \%$ is documented [3]. The DNN-value, based on 5 facial images per individual, was $91 \%$ for gay men. Hence, very similar results were obtained in two independent studies, based on different methods and non-overlapping samples of males $[3,6]$. These results support the same general conclusion, as summarized above. Although those studies were not designed to find evidence for the origins of erotic preferences, they are consistent with the "prenatal hormone theory" of sexual orientation, which states that same-genderattractions result from under (or over) exposure of the developing fetus to androgens, such as testosterone [7].

Finally, we want to mention that in Germany, according to the state-supported gender-ideology, being gay is regarded as a lifestylechoice based on the belief that "today a man wants to be straight, but tomorrow he may prefer to be gay". This idea is hardly compatible with the empirical findings summarized above $[6,7]$.

We think that, in some individuals, sexual orientation may in fact be a lifestyle-choice or a social construct. However, the science of sexual orientation seems to be clear: for most men, erotic attraction is not a matter of choice, but has a biological basis and is determined before birth. Recent data support the well-established correlation between prenatal hormone levels and feminized facial features in homosexual men [6,7]. However, there is clearly need for more research as to the origin and evolutionary consequences of homosexuality in humans.

\section{Summary and conclusions}

Based on the "Five-Factor-Model of Personality" (Openness, Conscientiousness, Extraversion, Agreeableness, and Neuroticism), computer-assisted analyses of thousands of Facebook-Likes showed that numerous personal attributes, such as ethnicity (race; Caucasian vs. African American), gender (male/female) or sexual orientation (gay vs. straight) can be predicted with accuracies of 88 to $95 \%$. Hence, at least some aspects of our character are expressed in our facial online presentations and behaviors. However, such data (digital footprints) should not be misused to denounce certain groups of people. All humans have equal rights, despite the fact we are all unique (un-equal) "genetic recombinants", as a result of the power of sexual reproduction (combined with germline-mutations), and random mating in natural populations [2].

Finally, we want to stress that the development of more powerful DNN algorithms with higher accuracy, the use of better images, and larger numbers of portraits per person may pose serious privacy threats to all of us. As pointed out previously, it is obvious that this post-privacy world of the future will be a much safer place if society is composed of tolerant, scientifically educated people who respect human rights and the dignity of all members of our diverse species $[2,6,7]$.

The significance of these findings for the occurrence of FacebookAddiction, mentioned in the Introduction is obvious [1]. This social media platform provides deep insights into the personality of other people. Since humans are "evolved social animals", the ability to inspect intimate personality traits of conspecifics fulfills a psychological need for knowledge about the feelings and mood of other people [2]. This desire to "switch into the brain of a foreigner" is so strong that Facebook-addiction may develop, a condition, which is difficult to overcome [8].

\section{Acknowledgement}

This article was designed and written during a stay at Stanford University/April 2018 of U. Kutschera (Graduate School of Business, Stanford University, 665 Knight Way, Stanford CA 94305, USA.) and is based on a discussion between U. K. and Prof. Dr. Michal Kosinski. We thank the Editors and anonymous Referees for constructive comments.

\section{Competing interests}

The authors have no competing interests.

\section{References}

1. Pathak P (2019) Facebook addicts bad at making decisions because they think like cocaine or heroin addicts: Study. 14: 1-2.

2. Kutschera U (2017) Evolution. Reference Module in Life Sciences. Article 06399, Elsevier Inc, 1-5.

3. Kosinski M, Stillwell D, Graepel T (2013) Private traits and attributes are predictable from digital records of human behavior. Proc Natl Acad Sci U S A 110: 5802-5805. [Crossref]

4. Youyou W, Kosinski M, Stillwell D (2015) Computer-based personality judgments are more accurate than those made by humans. Proc Natl Acad Sci U S A 112: 1036-1040. [Crossref]

5. Matz SC, Kosinski M, Nave G, Stillwell DJ (2017) Psychological targeting as an effective approach to digital mass persuasion. Proc Natl Acad Sci U S A 114: 12714 12719. [Crossref]

6. Wang Y, Kosinski M (2018) Deep neural networks are more accurate than humans at detecting sexual orientation from facial images. J Pers Soc Psychol 114: 246-257. [Crossref]

7. Kutschera U (2018) Darwin's nose: The revival of physiognomy at Stanford University Jpn J Med 1: 243-246.

8. Carey B (2019) This is your brain off Facebook. Nytimes.com/2019/01/30/health/ facebook-psychology-health.html: 1-5.

Copyright: (C2019 Kutschera U. This is an open-access article distributed under the terms of the Creative Commons Attribution License, which permits unrestricted use, distribution, and reproduction in any medium, provided the original author and source are credited. 